\title{
El mito del primer mundo. Capitalismo y barbarie*
}

\section{Nancy Ramírez Poloche}

\begin{abstract}
El sistema capitalista no es otra cosa que una vulgaridad criminal convertida en una abstracción. Es una simple operación matemática (robar al prójimo con la política y la guerra) que terminó convertida en una civilización: El sistema capitalista nivelado como "mundo único" a escala planetaria. Resumido, el capitalismo no es nada más que una empresa de ladrones comunes, que niveló a escala planetaria un "sistema" (económico, politico y social) para legalizar y legitimar con leyes e instituciones un robo masivo y planetario de trabajo social y de recursos naturales, disfrazado de "economia mundial"
\end{abstract}

Manuel Freytas ${ }^{1}$

La crisis económica que hoy perturba el equilibrio de Europa y del mundo tiene que ver, como es obvio, con la sostenibilidad del capitalismo. La deuda que han contraído con los bancos algunos países de la Unión Europea (UE) ha llevado a una errónea política de recortes en la inversión que ha afectado al ciudadano común y corriente, cuando es bien sabido que dicha crisis en cierta medida se ha generado por el mal manejo y la corrupción de los políticos de turno. En la UE estas medidas han dejado al descubierto un estado de debilidad de la economía europea y están golpeando duramente los derechos sociales de todos los ciudadanos. La recesión económica se acompaña de grandes empresas en crisis y de un desempleo rampante (apabullante) que acentúa el desequilibrio en la distribución de la riqueza: los pobres (mayoría) cada vez más pobres y los ricos (minoría) siempre más ricos. Estos factores son de sobra conocidos en Latinoamérica, pero es el mito del primer mundo el que se nos cae a pedazos. Sin embargo, los medios nos inundan de imágenes distorsionadas de lo que realmente está pasando en el Viejo Continente.

En la actualidad las instituciones democráticas están siendo sitiadas por fuerzas ocultas dirigidas a conquistar sectores claves de los gobiernos y a influenciar las decisiones políticas internas de los Estados, al punto de desplazar a su favor importantes recursos y determinar la naturaleza de los gobiernos. Estas fuerzas externas de élite no responden ante nadie y se niegan a someterse a cualquier proceso, creando así un enorme desequilibrio de poder entre las finanzas y la democracia. Al final el pueblo (que según los principios democráticos es soberano) ejerce solo un poder ficticio y los órganos de representación terminan regulándose por el desempeño del rating ${ }^{2}$ y "la dictadura del spread" 3 .

NANCY RAMÍREZ POLOCHE. Doctora en Antropología social y cultural. Universidad de Barcelona. Correo electrónico: nramirpo7@docd1. ub.edu.

* Artículo de reflexión derivado de la experiencia y trabajo etnográfico hecho por la autora durante mas de diez años de vivir en Europa donde realizó sus estudios de posgrado y ha hecho su vida profesional.

1. Manuel Freytas (2011). Es periodista, investigador, analista de estructuras del poder y especialista en inteligencia y comunicación estratégica. http://www.iarnoticias.com/2011/secciones/contrainformacion/0038_capitalismo_empr_criminal_27mayo2011.html

2. Método utilizado para evaluar los títulos de débito del Estado como los de las empresas con base en el riesgo financiero. Su valoración corre a cargo de las agencias del rating.

3. El spread es la tasa de rendimiento entre un bono u obligación bancaria y un título. Es también una operación financiera que combina activos financieros con el fin de obtener un valor determinado en un plazo preciso. 
Las graves dificultades que enfrenta la UE no pueden explicarse únicamente en función de la economía y las finanzas, las cuales están gozando de una atención casi exclusiva. No podemos soslayar el factor político-militar que cada día adquiere mayor peso y merece ser investigado en relación con la tempestad económica. Por encima de todo nos enfrentamos a un fenómeno muy actual que gracias a la globalización está produciendo unos efectos que en opinión de los expertos son muy diferentes a los previstos tanto por sus defensores como por sus críticos, sean estos de derecha o de izquierda. Esta crisis es lo suficientemente aguda como para optar por soluciones "milagrosas" y quien diga tener la receta para una rápida salida es solo un charlatán. Los problemas complejos no precisan de soluciones simples y si este desastre tomó 40 años en gestarse, no se puede esperar su disolución en pocos meses. Ello no significa que no haya nada que hacer, pero es obvio que Europa sufrirá una época de escasez.

Este artículo, más que proponer una fórmula salvadora busca reunir una serie de argumentos para ser discutidos. Se exponen ideas que se inspiran en diferentes perspectivas que van desde lo político a lo cultural y se parte de la premisa de que la economía no es una ciencia exacta, por lo cual debe ser ubicada en un contexto social y moral en el que sus planteamientos puedan ser cuestionados. En las decisiones económicas y políticas no se pueden ignorar los intereses en juego los cuales inevitablemente son opuestos, por lo tanto este escrito no intenta ser neutral. La crisis actual no es solo financiera, por lo que no requiere exclusivamente soluciones de orden monetario; de hecho, la crisis se está desplazando cada vez más al terreno de la política. Agudos procesos de desestabilización se enfocan hacia un cambio económico y político-social (en particular las revoluciones árabes que han llevado a una alteración de las condiciones que rigen el mercado del petróleo) y son vistos por muchos con evidente nerviosismo. Estos difíciles momentos que vive Europa no son los más adecuados para que los gobiernos consulten a los "señores de las finanzas" con la idea de hallar posibles soluciones a la crisis que ellos mismos han causado.

\section{El caso griego}

Grecia es un país que basa su economía en el turismo y hasta los años ochenta fue gobernado de manera eficiente. La entrada en la eurozona significó para su economía un importante desequilibrio, aunque el enorme déficit fiscal que vivía no era algo nuevo puesto que desde 1993 se habían dado graves casos de corrupción política. A partir del 2004 y hasta el 2009, el primer ministro Costas Caramanlís ${ }^{4}$ contrató durante su gobierno a más de 100 mil nuevos funcionarios a los que concedió sustanciosos incrementos salariales. $\mathrm{Su}$ administración se caracterizó por el descontrol del gasto lo que en últimas causó el deterioro de la economía griega. Sin duda, una de las causas de la actual crisis europea ha sido el mal manejo de la política económica griega ejercida de manera irresponsable y corrupta. Un claro ejemplo de ello fue el gran negociado de las pensiones, sacado a la luz cuando se descubrió que muchas de ellas correspondían a personas fallecidas que intencionalmente eran mantenidas en el sistema para así continuar con su cobro. Incluso se encontraron familias que cobraban tres y cuatro pensiones a las que no tenían derecho, lo que le costaba al Estado unos 550 millones de euros al año. Sus funcionarios gozan de la edad de jubilación más baja de toda Europa (cincuenta años para las mujeres y cincuenta y cinco para los hombres) en comparación con Francia donde la edad de pensión es de sesenta y dos ańos y se recibe en promedio el $51 \%$ del último salario; $y$ con Alemania que pensiona a los sesenta y siete años (hasta las reformas actuales los jubilados griegos recibían un $96 \%$ del último salario). Es claro que los griegos tienen un estándar de vida más alto del que se podrían permitir; sin embargo, las escandalosas políticas de recortes de los derechos sociales han tenido que enfrentarse a la ira y a la resistencia más aguda de los trabajadores y sectores populares.

Tras la derrota electoral de Caramanlís en octubre de 2009, el nuevo Primer Ministro Yorgos Andreas Papandréu ${ }^{5}$ se encontró con la enorme brecha entre las cifras oficiales y las reales. Durante los primeros años de su gobierno intentó tapar el agujero fiscal y saldar la vertiginosa deuda, pero

4. Líder del partido Nueva Democracia, nombrado Primer Ministro de Grecia en el 2004. Su gobierno duró hasta el 2009 cuando tuvo que convocar elecciones anticipadas debido a la crisis económica.

5. Presidente del Movimiento Socialista Panhelénico. Primer Ministro de Grecia desde el 2009 hasta el 2011 cuando dimitió debido al empeoramiento de la crisis económica. 
como era de esperarse la crisis se trasladó del sector privado al sector público, es decir, al Estado. El déficit fiscal y la deuda pública afectaron al sector más vulnerable de la economía griega y trasfirieron el problema a otros ámbitos. Se iniciaron los recortes de nómina y la eliminación de los privilegios a los funcionarios públicos y a otros trabajadores a fin de corregir la maltrecha situación económica del país, pero esto desató unas serie de huelgas y violentas protestas.

En el 2011 la deuda pública griega se disparó por encima del referente alemán y obligó al gobierno a recurrir a la ayuda del Fondo Monetario Internacional y al Eurogrupo para evitar la bancarrota estatal, lo cual le significó entregar parte de su soberanía y someter sus cuentas internas al escrutinio internacional. El Banco Central Europeo (BCE) aprovechando la desventura de los débiles, en una operación sin precedentes compró bonos y títulos de aquellos socios de la eurozona que tuvieran la deuda más delicada. En la actualidad Grecia, es un país reducido al extremo, dirigido por la especulación, sometido a grandes sacrificios y chantajeado por la amenaza de una salida devastadora de la eurozona que sería fatal para su economía. En estas circunstancias fue elegido en el año 2012 Antonis Samaras ${ }^{6}$ como Primer Ministro. Líder del partido Nueva Democracia y máximo exponente de la derecha griega, llegó al poder bajo sospecha de fraude electoral con tan solo el $29.66 \%$ de los votos. Samaras ha debido hacer un gran esfuerzo para lograr un acuerdo tanto interno como con la troika de prestamistas: la UE, el BCE y el FMI. Las reformas propuestas por Samaras apuntan a la reestructuración de los servicios públicos y la presión que ha recibido de los países de la eurozona pone en evidencia que lo importante no es salvar al pueblo griego de la crisis sino el pago puntual a los bancos prestamistas internacionales. Los máximos líderes de la eurozona (Alemania y Francia) han dejado en claro el mensaje al Primer Ministro, quien se ha lanzado a la ofensiva con su plan de reformas y recortes de los derechos sociales del pueblo griego. Algunos políticos en Alemania y otros países han sugerido que Grecia, con su alta carga de deuda y ahora en su quinto año de recesión, estaría mejor fuera de la eurozona. La crítica situación de Grecia la ha llevado al punto de proponer el alquiler de cuarenta islas deshabitadas, a lo que Israel respondió con un ofrecimiento de compra.

Samaras por su parte, ha emprendido un plan de recortes presupuestarios para poder cumplir con las exigencias externas. Las manifestaciones y protestas del pueblo griego están a la orden del día, pues los recortes en los sueldos, las bajas en las pensiones y el desempleo golpean a millares de familias. Las manifestaciones intentan presionar al gobierno y a los políticos, pero las medidas avanzan con el argumento de mantener solvente al Estado. Las propuestas de la UE y el FMI son las de recortar sueldos, indemnizar por despidos y descartar las alzas automáticas de salarios. La violencia policial para impedir las manifestaciones no se hizo esperar. Al parecer, en lo único que todavía se hacen grandes inversiones es en reforzar el aparato policial, adquirir más armamento y aumentar el pie de fuerza para controlar la furia de miles de griegos que lo han perdido todo. Enfrentados al hambre y a la precariedad económica, lo único que les queda es levantar su voz para ser escuchados.

Es así como hemos llegado a la paradoja de que toda Europa, incluso aquella que dice llamarse de izquierda, ha celebrado la victoria de la derecha en Grecia. Se trata, como es evidente, de un engranaje en el que los Estados de la UE así como el parlamento y los gobiernos elegidos por los ciudadanos, se han convertido en una rueda mortal dirigida por los oscuros intereses de los bancos. Noam Chomsky ha definido estas fuerzas escondidas como una especie de "senado virtual" cuyos integrantes, hombres sin escrúpulos aconsejados por las agencias de rating, determinan las políticas nacionales. Cuando consideran en peligro sus intereses, los inversionistas amenazan con trasladar el capital y poner en peligro la estabilidad económica de los países. De esta forma, en la actualidad cuenta más el juicio de los mercados que el voto popular; la opinión de los organismos financieros que la mayoría de un gobierno democrático. La razón pública está constreñida por un grupo anónimo y se vive un fenómeno de privatización de la democracia.

Cuando se habla de crisis económica en la UE a menudo permanece oculto un aspecto en verdad preocupante como lo es el proceso de reducción

6. Licenciado en economía en Amherst College en el año1974. Estudió en Harvard University en el año 1976 y fue Ministro de Economía en 1989. 
del espacio democrático y del poder público frente al poder financiero de los bancos y de las empresas multinacionales privadas. Queda claro que las grandes fusiones bancarias han dado lugar a la formación de una superaristocracia financiera que en gran medida altera la relación entre el poder económico y el poder político y deja atrás la imagen de los bancos como simples administradores de liquidez.

Se ha llegado a establecer en el imaginario colectivo que los ladrones y asesinos abundan entre los estratos más bajos, pero dado el tamaño extremadamente pequeño de la nueva superaristocracia financiera parece que la propensión a la delincuencia de muchos financieros es mayor que en cualquier estrato y de hecho se puede afirmar que la actual crisis económica es producto de la avaricia desmedida de los mercados financieros. El sistema financiero es potencialmente criminal, sobre todo si se tiene en cuenta que no existen leyes lo suficientemente estrictas y claras que garanticen una protección contra sus abusos. Está claro que los gobiernos nacionales no deciden el ingreso de inversión extranjera en sus países; son los inversores quienes lo hacen de acuerdo con sus propias reglas. La superaristocracia financiera es la manifestación más perfecta del poder irresponsable en contra de la democracia.

La distribución de la riqueza no es solo una cuestión moral sino también política, porque afecta la cuota de poder social de cada grupo o clase. Obviamente, en una sociedad tecnológicamente avanzada con una gran masa de mercancías de alto valor, el reparto de la riqueza puede tomar distribuciones de diverso tipo: en forma de $\mathrm{U}$ con un sesgo fuerte en ambos extremos; de arco liso con una sucesión continua de puntos de la riqueza; o una curva de Gauss ${ }^{7}$ con una amplia gama de medio múltiples y pequeñas minorías a los extremos. Cada curva propone un modelo social diferente con una distribución distinta de la riqueza y el poder. En una sociedad desarrollada, la determinación del umbral de la riqueza no es dada por el consumo sino por la acumulación de riqueza. Esta sin un equivalente real no mueve la producción mundial, pero es mediante la transferencia de ingresos como se crea una participación significativa para financiar la economía real y por lo tanto es esta superaristocracia financiera la que gana cuotas importantes en el poder social. Ello significa que en la actualidad se entrega el poder económico a unos pocos para disponer de la vida de millones de personas en el planeta.

\section{La Italia de Berlusconi}

En el otońo de 2008 se lanzaron a las calles en Italia dos generaciones. Por primera vez en una protesta social se unieron padres e hijos en pos de un objetivo común. Las madres, con sus niños de la mano, caminaban decididas detrás de las grandes pancartas de los partidos políticos y los sindicatos; madres que compartían con sus hijos una condición general que en dos décadas se había agravado, como era la condena a vivir en peores condiciones sociales que las generaciones anteriores. Un estudio realizado por el Istat de Italia (Instituto Nacional de Estadística) en el 2009 sentenciaba que "[...] la permanencia de los jóvenes en el hogar de la familia es uno de los principales problemas de Italia”. El estudio, publicado con el elocuente título de Dificultades de los jóvenes en la transición a la edad adulta y rutas críticas de la vida las mujeres, expuso con tono preocupante la urgencia de la participación del gobierno en la solución de las situaciones de emergencia en las que estaban inmersas las nuevas generaciones. Otro fenómeno inquietante era el abandono del hogar de más de la mitad de los jóvenes italianos, no por haber conseguido un trabajo satisfactorio sino porque se casaban ${ }^{8}$, como era la costumbre hace un siglo. La permanencia de los jóvenes por demasiado tiempo en la casa de sus padres es el producto de la conjunción de una serie de factores económicos que deben ser afrontados por toda la sociedad italiana. Se podría afirmar que Italia no es un país para los jóvenes. Estos están hartos de escuchar hablar

7. La curva de Gauss es utilizada en estadística y probabilidad. La campana de Gauss es una representación gráfica de la distribución normal de un grupo de datos.

8. Tal vez el matrimonio no sea la única salida para el creciente ejército de jóvenes que no pueden liberarse del yugo de la inseguridad que los invade después de su graduación. Pero lo cierto es que llegar a ser económicamente independiente y fundar una familia solo por el trabajo se está convirtiendo en un problema. Hoy en día, uno de cada tres jóvenes menores de 40 años puede lograr un contrato indefinido en su primer intento. Los otros dos son contratados bajo la modalidad de un contrato a corto plazo u otros contratos atípicos. 
del "mammismo" y del "bamboccionismo"' términos que hacen parte del argot de los políticos de turno y de los medios de comunicación a fin ocultar la verdadera situación de crisis producto de los cambios económicos y políticos llevados a cabo desde la década de 1990 y que han reducido de manera importante los derechos sociales. Los jóvenes italianos sufren una ausencia de representación política y sindical que les impide elegir un gobierno que responda a sus necesidades urgentes. Esto es evidente y contrario a la afirmación sensacionalista del Istat en el sentido de que el "bamboccionismo" es un fenómeno cultural o antropológico. Se trata de un drama colectivo que tiene raíces sociales y económicas que deben ser reconocidas. Italia no parece ser capaz de entender plenamente que el llamado "bamboccionismo" y el "mammismo" son solo el síntoma más alarmante de un país bloqueado que ha cambiado profundamente desde los años ochenta. Sin duda, los doce años de mal gobierno de Silvio Berlusconi han dejado a Italia en la quiebra y en manos de un gobierno técnico que ha tenido la responsabilidad de ejecutar las duras medidas económicas que hoy tienen a miles de italianos en la ruina económica. En agosto del 2011, el gobierno Berlusconi apoyado en el artículo 8 de la Ley de Hacienda eliminó el contrato de trabajo nacional que permitía acuerdos empresariales sobre los derechos fundamentales de los trabajadores alusivos a la clasificación y calificación del personal, a las horas de trabajo, a los contratos terminados, al tiempo de los contratos, al sistema de solidaridad, al uso de la oferta de trabajo y al modo de aplicación.

Desde los ańos noventa hasta el día de hoy conseguir un trabajo fijo a tiempo indefinido en Italia es toda una odisea. Con la entrada en la UE el país ha tenido que adaptarse a una fuerte presión externa que ha empeorado las condiciones laborales con contratos flexibles, razón por la cual el llamado Estatuto de los Trabajadores no ha podido hacer nada contra la ley de los despidos sin justificación. Los sindicatos, conformados actualmente por unas pocas decenas de miles de trabajadores atípicos, y los pensionistas que se cuentan por millones, han emprendido una lucha tenaz contra el gobierno por los atropellos que generan estos contratos laborales que no ofrecen la más mínima garantía a los empleados. La flexibilidad de la mano de obra es una propuesta impuesta que solo ha dejado tras de sí una mayor tasa de desempleo y millones de trabajadores con un precario contrato temporal y saltando de un trabajo a otro sin ninguna garantía, un problema que se ha vuelto especialmente delicado durante esta reciente recesión. La crisis económica ha cobrado sus víctimas especialmente entre los jóvenes. Mientras cientos de miles de puestos de trabajo con contrato a tiempo indefinido fueron congelados, la gente fue enviada a la Caja de Integración pero después no se les renovó el contrato. La Caja de Integración pasará a la historia como otra pérdida de un derecho social.

El gobierno italiano ha promulgado leyes para regular los contratos atípicos, pero con objetivos tan estrechos que su provecho es para pocos. Los futuros desempleados se quedan en casa con cero ingresos, por eso las declaraciones de algunos políticos italianos son totalmente desfasadas. La Ministra de Trabajo Elsa Fornero ${ }^{10}$ ha dicho recientemente que: "[...] los jóvenes italianos no puedan permitirse ser quisquillosos ${ }^{11}$ cuando se trata de acceder al mundo del trabajo". Esta afirmación levantó una polvareda en toda Italia, puesto que es muy fácil para una ministra del trabajo hacer declaraciones del tipo "el trabajo no es un derecho y debe ser ganado". En realidad, estas afirmaciones son banalizaciones que distraen de la dura realidad que viven no solo los jóvenes sino sus padres. Por su parte, los medios de comunicación hacen comentarios insultantes sin argumentos serios ni sustento estadístico alguno que solo contribuyen a fortalecer los prejuicios y a construir una realidad

9. Estos términos hacen referencia al culto a la madre en una especie de dependencia patológica. Así mismo, se relacionan con la incapacidad de madurar de los jóvenes italianos y de asumir sus propias decisiones, generando con ello una especie de infantilismo.

10. Profesora desde 2000 de Economía de la Facultad de Economía de la Universidad de Turín. Enseña Macroeconomía y Economía de ahorro, jubilación y fondos de pensiones. Su investigación científica es referente a los sistemas de protección social, la reforma de pensiones públicas y privadas, el envejecimiento de la población y las opciones de jubilación, ahorros y seguros de vida. El 16 de noviembre 2011 fue nombrada Ministro de Trabajo y Asuntos Sociales con la responsabilidad de igualdad de oportunidades del gobierno de Monti. En la historia de la república italiana es la segunda mujer después de Tina Anselmi, en presidir este ministerio.

11. Giovani, per la Fornero sono choosy: la polemica non si placa 26/10/2012 http://www.controcampus.it/2012/10/giovani-per-lafornero-sono-choosy-la-polemica-non-si-placal 
distorsionada y presuntuosa que solo conduce a la demagogia ${ }^{12}$.

La respuesta a la ministra Fornero por parte del movimiento estudiantil italiano es clara y elocuente, pues invita a dejar la arrogancia y a reflexionar sobre lo que está ocurriendo en el país:

Estamos sorprendidos por la retórica de la ministra que utiliza los mismos métodos de comunicación de la astucia política, para corregir el tono después de una metedura de pata evidente, pero no se preocupa en absoluto por vincular la universidad con el mundo del trabajo ni organizar un plan nacional de financiación para las pequeñas empresas, o un plan de integración de NEET (jóvenes que no estudian ni trabajan) en el sector de la artesanía que ofrezca oportunidades de empleo. Si bien vemos una lágrima hipócrita en representantes y representados, -sobre todo en un gobierno que tiene que lidiar con los estándares europeos y la calidad de vida de su pueblo-, algunos de estos comentarios se deben evitar, ya que generan el odio social y la sospecha del sistema político que los representa. Hay que recordar que el colegio técnico es el único elemento de movilidad social en Italia, por lo que puede ser humillante recibir estos insultos después de los sacrificios y todo porque la política italiana no maneja el mercado de trabajo o porque no es capaz de crear un sistema universitario que pueda seguir el ritmo de los tiempos ${ }^{13}$.

El gobierno "técnico" de Mario Monti ${ }^{14}$, con la reforma "Fornero", derogó la norma que ordenaba la restitución del trabajador despedido sin justa causa, lo que va en contra de una sentencia del juzgado de lo social a favor del trabajador. Los primeros despidos ya se han hecho y esta nueva reforma "Fornero" es la que está afectando duramente a los jóvenes italianos. Una característica distintiva de la actual legislación laboral es prefigurar relaciones sociales autoritarias que afectan la vida de todos los ciudadanos respetuosos de la ley, lo cual viola un derecho fundamental como es el derecho al trabajo. El resultado son los atípicos contratos laborales que condenan a los jóvenes italianos a una especie de limbo de "mil euros" y sin posibilidad alguna de hacer una carrera; los jóvenes que ingresan al mundo del trabajo lo viven de manera dramática. En este sentido, el reciente informe Eurofound ${ }^{15}$ (Fundación Europea para mejora de las condiciones de vida y de trabajo) publicado en el 2012 expone la preocupación de los gobiernos de la UE en relación con la población joven entre los quince y veintinueve años (94 millones). Esta generación que vive una era de globalización total tendrá que hacer frente a la responsabilidad de un envejecimiento, por lo tanto es asunto de gran preocupación el hecho de que hayan sido golpeados tan duramente por la crisis económica (solo el $34 \%$ estaban empleados en el 2011, la cifra más baja jamás registrada por Eurostat). El desempleo en los jóvenes aumentó en 1.5 millones, hasta alcanzar los 5.5 millones (21\%) en el 2011. Según Eurostat, en el 20117.5 millones de jóvenes entre los quince y los veinticuatro ańos y 6.5 millones entre los veinticinco y los veintinueve ańos fueron excluidos del mercado de trabajo y de la educación en Europa. Esto corresponde a un aumento significativo en la tasa NEET: en el 2008 estas cifras eran del $11 \%$ y del $17 \%$ respectivamente. Hay una variación enorme entre los Estados miembros, con índices que van desde el $7 \%$ (Luxemburgo y los Países Bajos) y más del $17 \%$ (Bulgaria, Irlanda, Italia y España). Algunos jóvenes están en mayor riesgo de ser NEET que otros.

Las personas con bajos niveles de educación tienen tres veces más probabilidades de ser NEET en comparación con aquellos que tienen una educación terciaria y los jóvenes de origen inmigrante tienen un $70 \%$ más de probabilidades de convertirse en NEET que los nacionales. Los jóvenes que sufren de algún tipo de discapacidad o problemas

12. Este mecanismo es común en Latinoamérica y sobre el cual podemos citar numerosos casos en Italia. Un ejemplo reciente es el de los "falsos inválidos" que estaban cobrando pensiones sin tener ningún tipo de discapacidad. Son casos aislados que desafortunadamente han sido usados para quitar ayudas a los servicios sociales y a los inválidos verdaderos. Repite una mentira mil veces, por lo que termina siendo una verdad. Este juego político se argumenta en Italia con generalizaciones sin ningún estudio o investigación previos, pero sirve de argumento para convencer a la opinión pública. Se basan en comentarios fútiles como decir que un profesor es perezoso o sin preparación y tiene un montón de vacaciones, para inmediatamente proponer cargas de trabajo impensables.

13. http://www.controcampus.it/2012/10/litalia-dei-choosy-lopinione-del-movimento-studentesco-nazionale/

14. Economista, académico y político Italiano. Senador desde el 9 de noviembre de 2011. Presidente de Italia y exministro de Economía.

15. Este informe analiza la situación del mercado laboral de los jóvenes en Europa con un enfoque específico en el grupo categorizado como NEET. En él se examinan los determinantes de la pertenencia al grupo NEET, y sus costos económicos y sociales. Además, se evalúa la política de los Estados miembros de la Unión Europea y qué intentos han hechos estos para apoyar a los jóvenes en el mercado de trabajo. 
de salud tienen un $40 \%$ más de probabilidades de ser NEET que los de buena salud. En relación con esto Bauman afirma:

No soy un profeta, pero puedo mirar a mi alrededor y veo pocas señales de que estemos en el camino para salir de la crisis. Este colapso del crédito ha sido al final una redistribución de riqueza que ha dado más a unos pocos ricos y ha hecho más pobres a los pobres, especialmente a los jóvenes. Un $52 \%$ de ellos está sin empleo y eso es muy grave; no pasaba algo así desde la Segunda Guerra Mundial. Y eso los frustra, porque les quita su dignidad haciéndoles sentir que nadie los quiere, que no sirven para nada. Se está quitando riqueza a los más débiles y eso es muy peligroso; y los gobiernos sienten la presión de los bancos y de las instituciones financieras. $Y$ ante esto no hay caminos intermedios: o te rindes a lo que te piden los bancos y la economía, haciendo más fácil la vida a los bancos y penalizando a los débiles; o defiendes los intereses de tu población ${ }^{16}$.

La crisis económica devora los recursos familiares incluidos aquellos destinados a proteger y asegurar el futuro de los jóvenes. No es cierto, como pretende mostrar el informe Eurofound, que estudiar una carrera garantiza el futuro laboral de los jóvenes. Son cientos los graduados que no encuentran empleo, por tal razón muchos prefieren no realizar estudios al ver a sus compañeros profesionales en la misma situación de paro. Lo anterior ha llevado al derrumbe de una de las utopías más populares: la de la universidad al alcance de todos. Italia cuenta con algunas de las universidades más antiguas del mundo, pero después de la reforma Berlinguer ${ }^{17}$ sobresale una increíble cantidad de sillas vacías que costaron millones de euros y que pudieron utilizarse para la investigación o la docencia, pero asombrosamente sirven de asiento a cuatro o cinco estudiantes que asisten a cursos con nombres a menudo grotescos. En el último informe elaborado por el Instituto de los Estados se muestra cómo un $63.3 \%$ de los estudiantes italianos acusa a los maestros de incapacidad para relacionarse con ellos. Los datos referidos al 2004 revelan que el $50.5 \%$ opina que sus profesores son aburridos, uno de cada tres (37.1\%) juzga que carecen de las competencias necesarias con respecto a la materia enseñada y cerca de un tercio considera que sus profesores desconfían de la innovación tecnológica. Un detalle interesante estriba en que la percepción de la docilidad excesiva se ha incrementado en casi un $10 \%$ con respecto a 1984 que fue del $17.9 \%$. Por otra parte y en contradicción con las expectativas más de dos tercios $(68,1 \%)$ considera que la educación escolar es importante.

Con respecto al 2003, Mario Draghi ${ }^{18}$ recordó los datos ahora dramáticos en relación con la escuela y la universidad. En Italia, solo un tercio de las personas entre los 25 y los 64 años se graduó y una de cada diez posee un grado, en comparación con el promedio de los países de la Ocde (Organización para la Cooperación y el Desarrollo Económico) cuyo porcentaje es del $41 \%$ y del $24 \%$ respectivamente. Sin embargo, el problema no radica solo en el abandono escolar sino en la mediocre calidad de los resultados obtenidos en la escuela.

Desde hace más de dos años las medidas introducidas por la Ministra de Educación Mariastella Gelmini ${ }^{19}$ fueron dictadas más que todo por la necesidad de reducir el gasto público. Los recortes fiscales aplicados a la educación buscan principalmente la racionalización de los gastos $\mathrm{y}$ sus objetivos se concentraron en la consolidación de 350 instituciones y en la reducción de 42.000 docentes que suponían para el Estado italiano un ahorro de mil seiscientos millones de euros para los años 2009-2010 y de tres mil doscientos millones en los siguientes tres años. Los grandes recortes de personal afectaron principalmente a Campania (6.100 profesores), Sicilia (5.000), Lombardía (4.800) y Puglia (4.000), sin duda una reorganización draconiana que en tres años

16. Zygmunt Bauman. "Nos han impuesto que eres más feliz cuanto más consumes y más compites" 29/10/2012. http://sociologosplebeyos.com/2012/10/29/nos-han-impuesto-que-eres-mas-feliz-cuanto-mas-consumes-y-mascompites-zygmunt-bauman/

17. Una de las críticas más enérgicas a esta reforma era que imposibilitaba el continuar los cursos de formación si se tenía una licencia en educación media. La reforma Berlingue no hablaba literalmente de abandono escolar pero sí de una escuela-trabajo. El mismo decreto imponía una obligación en formación profesional hasta los 18 años al cabo de los cuales se debía conseguir el diploma.

18. Economista, presidente del Banco Central Europeo y gobernador de la banca italiana.

19. La Ley Gelmini comúnmente indica el conjunto de actos legislativos relativos al sector de la educación que entró en vigor durante el mandato de la consejera de educación, universidades e investigación Mariastella Gelmini. Estas medidas están contenidas en algunos artículos de las leyes 133/2008 y 169/2008, cuyo principal propósito es reformar todo el sistema educativo italiano. La reforma entró en vigor el primero de septiembre de 2009 para los niveles primario y secundario y para la escuela secundaria lo hizo el primero de septiembre de 2010. La Ley 240/10 del 30 de diciembre para reformar el sistema universitario entró en vigor en enero del 2011 
condujo al éxodo de 133.000 docentes y a un recorte de cerca de seis millones de euros cuyo resultado fue un dramático descenso en la calidad de la educación de las escuelas italianas. Según la Ocde (Organización para la Cooperación y el Desarrollo Económico) ahora Italia figura en el puesto 26 entre los 29 países industrializados. Es sin duda un mal momento para proponer un plan de recortes económicos y si ello es imposible debe hacerse selectivamente y no de una manera abrupta que solo busque proteger las necesidades de las cuentas públicas. Si es el ministro de Finanzas quien propone reformar la escuela en Italia es porque algo no funciona.

Una imagen de una universidad libre y justa si bien lejana sigue siendo defendida en las calles en nombre del derecho a la educación para todos. No obstante, el $20 \%$ de estudiantes abandona la universidad después del primer año y solo un $45 \%$ obtiene el grado, en comparación con el $69 \%$ de la Ocde. Las becas y otras formas de financiamiento para los estudiantes de las universidades italianas se convirtieron en un lujo solo al alcance los hijos de los ricos, subvencionado incluso con los impuestos de los pobres. A todo el panorama anterior se suman los escándalos de corrupción de profesores y rectores que han puesto en marcha una especie de "sistema señorial" que no tiene nada que ver con la educación en sí misma pero que ha montado un método de captación de nuevos reclutas basado en la "esclavitud académica" o la relación familiar puesto en evidencia por los escándalos en las universidades de Messina y Bari. Otro problema se refiere a las carreras académicas cuya calidad educativa se basa en la antigüedad del profesor en la institución más que en el mérito; aún más, los profesores e investigadores más jóvenes están mal pagados en comparación con otras universidades europeas o americanas (a los profesores más antiguos se les paga la jubilación en peso oro, una progresión salarial mucho más rápida que en otros países, pero sobre la base de años de trabajo y no de publicaciones u otros índices de mérito). El resultado es que en los EE. UU la proporción entre los salarios típicos de investigadores jóvenes y auxiliares es de 1.5 a 1 mientras en Italia la brecha alcanza la increíble proporción de 4.5 a 1 .
La escuela italiana presenta otro inconveniente y es el envejecimiento del cuerpo docente. La Fundación Giovanni Agnelli en un estudio llevado a cabo sobre la escuela en Italia en el 2009 estima que en la próxima década cerca de 300.000 profesores dejarán la enseñanza al haber alcanzado el límite de edad; hoy los profesores italianos están entre los más viejos del mundo y su edad promedio es de más de cincuenta años. El rango de edad es hasta los cuarenta y siete en la escuela primaria y hasta los cincuenta y uno en el primer ciclo de secundaria y media superior (la edad promedio es de más de cincuenta y tres años); así, los profesores con más de cincuenta años son más del $55 \%$ del total. Esta situación no es propia de ningún otro país europeo. En el Reino Unido por ejemplo, los docentes con más de 50 años son solo el $32 \%$, en Francia son un 30 \% y en España el 28 \%. La práctica de conseguir trabajo en la escuela pública con un sueldo de hambre pero con un contrato fijo con el fin de lograr una pensión ya no funciona. Los profesores italianos de secundaria están entre los peor pagados de Europa, su condición laboral es cada vez más precaria y su labor se desarrolla en estructuras físicas deterioradas y obsoletas.

Italia vive hoy otra tragedia que permanece oculta y es la de las mujeres. Menos de la mitad trabajan pese al objetivo trazado por el Tratado de $\operatorname{Lisboa}^{20}$ de un $60 \%$ para el 2010 . No basta una intención discursiva política para que un propósito se convierta en algo concreto. Las políticas de nivelación son prácticamente inexistentes y los preceptos sociales consideran que al dejar a las mujeres en casa estas van a tener más hijos. Pero han tenido que rendirse ante la amarga realidad: en promedio, cada mujer italiana tiene solo un hijo (1.3 para ser exactos). El politólogo italiano Maurizio Ferrara ha resumido bien la situación: "mujeres en el hogar, cunas vacías", situación que Emma Bonino $^{21}$ ha caracterizado como una "rebelión silenciosa" de las mujeres italianas. Mientras que las políticas efectivas de conciliación han elevado la tasa de natalidad en Francia y en Alemania, en Italia el promedio se ha estancado hace dos décadas en un hijo por mujer. Sin embargo, cabe destacar el aporte de las mujeres inmigrantes en el aumento

20. El Tratado de Lisboa fue firmado el 13 de diciembre del 2007. Entró en vigor el primero de diciembre del 2009 y todos los Estados miembros de la UE lo ratificaron mediante un procedimiento que variaba según el ordenamiento constitucional de cada uno de ellos.

21. Política italiana, vicepresidenta del Senado de la Republica desde 2008, es una de las representantes del radicalismo liberal. Ha estado ministra de comercio internacional, miembro de la organización para la prevensión de conflictos en el mundo. 
de la tasa a la natalidad sin el cual la situación sería aun más dramática. Cada madre extranjera tiene en promedio el doble de hijos de una madre italiana. Las paquistaníes y las egipcias alcanzan un pico de 5.5 y las mujeres de Túnez un 4.5; no obstante, es una tasa insuficiente si lo que se busca en general es asegurar un futuro para el país. Paradójicamente, es sobre las madres inmigrantes que recae la tarea de hacer frente a la falta de servicios sociales y a la carencia de jardines infantiles. Incluso cuando se aborda el problema de las diferencias de sexo en relación con la protección social o laboral, ellas ocupan la parte inferior de las estadísticas tanto a nivel europeo como de la Ocde.

En los últimos años, son los gobernantes tanto de izquierda como de derecha los que han tenido una gran responsabilidad en la falta de políticas de nivelación, lo cual está transformando a Italia en un país con pocos niños y con más de la mitad de las mujeres desempleadas. Un hecho que pesa sobre el futuro de Italia es el desequilibrio generacional que en veinte años amenaza con transformar a la nación en un país de cabezas blancas. Por cada niño hay dos personas mayores lo que significa que por cada trabajador joven habrá dos dependientes jubilados, una estadística que refleja la imagen de un país poco saludable. Quien sea el próximo primer ministro tendrá que cambiar las políticas iniciadas por Mario Monti.

Maurizio Landini ${ }^{22}$ considera que las políticas de Monti no han sacado a Italia de la crisis económica; por el contrario, han agravado aún más la crisis social. Si no se ponen en tela de juicio las causas que han producido la crisis no habrá manera de salir de ella lo cual se traducirá en una carnicería social. Se debe erigir un nuevo modelo de desarrollo que respete el medio ambiente y los derechos humanos y para ello se requiere una política industrial coherente con los tiempos, potenciar la investigación y la innovación y eliminar las desigualdades, la discriminación y la exclusión del trabajo de generaciones enteras de jóvenes. El dinero se debe buscar donde están los ingresos más altos y no recortando la educación, la cultura y la salud.

\section{España y los indignados}

La dinámica de la crisis económica española es muy parecida a la de Italia y Grecia. El efecto negativo se ha sentido sobre todo en la estructura social y en el empeoramiento de las condiciones de vida. Para el 2012 la crisis ha socavado el $18,8 \%$ del total de empleo previo a ella, y anulado el creado por la construcción o la llamada irónicamente "burbuja del ladrillo". Sin duda alguna, la especulación inmobiliaria favorecida por una legislación sobre el suelo excepcionalmente laxa en materia de vínculos ha sido el símbolo del "falso bienestar" de la España democrática. Se hace evidente la pésima gestión de las entidades financieras en relación con la política de créditos y el sector inmobiliario. Sin vacilación alguna se puede afirmar que esta crisis ha puesto en evidencia la fragilidad de un modelo de desarrollo basado en la construcción inmobiliaria.

Durante el 2012 el mercado laboral español eliminó 850.000 empleos y generó solamente 374.000 por regulación de empleo. La tasa española de paro se elevó al $26 \%$ y el desempleo juvenil es del $55 \%$ que incluye unos 150.000 jóvenes entre los veinticinco y los veintinueve años, los cuales emigraron al exterior iniciando así una diáspora de capital humano cualificado. En la actualidad, el total de la deuda española asciende a 4,3 billones de euros (un $402 \%$ del PIB) de los cuales un $19,1 \%$ corresponde a deuda pública y el $74.5 \%$ restante atañe a empresas privadas y entidades bancarias. Sin embargo, el total de la deuda debe ser pagada por todos los españoles sin tener en cuenta que son las grandes empresas, las entidades bancarias y los grandes patrimonios los que han defraudado al Estado español. Según datos del Sindicato de Técnicos de Hacienda, en el 2010 esta expoliación alcanzó la cifra de 42.711 millones de euros (un $71.8 \%$ del fraude fiscal total); no obstante, el gobierno español (llámese socialista o de derecha) ante estos hechos solo se ha dedicado al recorte de servicios sociales básicos a la ciudadanía.

La salida de la crisis se aventura lejana y el escenario de la poscrisis se puede situar en el 2017 tras una gran recesión traducida en más de una década perdida y una idealización social

22. Sindicalista italiano, Secretario General de la Federación de Empleados Operarios Metalúrgicos (FIOM). 
sellada con un incremento sin precedentes en la reciente historia española de las desigualdades sociales. Entre tanto, cunde la indignación y las protestas, pero no se vislumbra aún una alianza entre la izquierda social y los sectores críticos duramente golpeados por la recesión que ofrezca una alternativa transformadora. Dentro de este panorama ha surgido el llamado movimiento de los indignados que comenzó a tomar fuerza a partir de sus primeras manifestaciones pacíficas en mayo del 2011 con la toma de varias plazas emblemáticas en toda España para denunciar y mostrar el rechazo a un sistema político que se ha caracterizado por sus mentiras, abusos de poder y corrupción. El nombre de indignados se inspiró en la obra de Stéphane Hessel ${ }^{23}$ de 2010 llamada Indignezvous.

Los indignados no son un grupo homogéneo. Está conformado por gente de todas las generaciones entremezclados de una manera que no se compadece con la dinámica ordinaria de una sociedad de consumo que tiende a mantener separados los potenciales nichos del mercado. La opción básica en favor de la no violencia ha sido respetada con rigor a pesar de la brutalidad policiaca. En el tiempo transcurrido desde las primeras acampadas de los indignados en toda España el interés mediático ha disminuido notablemente, algo previsible que no marca puntos a favor ni en contra del movimiento. Previsible también fue el intento de criminalizar las protestas no violentas apostando a la posibilidad de producir manifestaciones violentas apelando a policías infiltrados a fin de desprestigiar el movimiento. Los indignados persiguen el sueño de una afirmación de la libertad y la autonomía y de la satisfacción de las necesidades primarias (salud, trabajo, vivienda, alimentación, educación) sin caer en la cuenta de que el capitalismo no tiene la capacidad de hacerlo aun extendiendo al infinito su entramado de interdependencias. El movimiento de los indignados implica poner en discusión el modelo económico y de desarrollo para lograr una mayor autonomía de los productores impulsando con ello la cooperación e incluso la desmonetarización de ciertos servicios.
En el discurso de los indignados se expone la urgente exigencia de lo que ellos denominan una "democracia económica directa" que postule una forma de control ciudadano sobre los recursos y excluya explícitamente el influjo estatal sobre los precios. Se trata de aspiraciones generosas que no alcanzan incluso para brindar una alternativa orgánica al sistema actual de financiación de la economía que salvaguarda los intereses de una banca privada que en tiempo de crisis recupera sus pérdidas gracias a las inyecciones de dinero público y a la restricción del gasto social. Los indignados siguen manteniendo cierta presencia en diversas plazas en toda España a pesar de que la ocupación del espacio público se vuelve cada día más difícil. Más prometedor parece ser el activismo desplegado por las distintas asociaciones de las víctimas de las hipotecas, en especial por la plataforma de afectados por la hipoteca $(\mathrm{PAH})^{24}$. La oposición a los desahucios es una causa popular que puede contribuir al arraigo del movimiento en las barriadas populares, sobre todo cuando sus promotores plantean la defensa vigorosa de cientos de personas que están perdiendo sus hogares. Entre los años 1998 y 2007 el número de hipotecas formalizadas anualmente fue de 822.000 , más de ocho millones en total. La lucha de la PAH desde que empezó la crisis en el 2007 se fundamenta en más de 350.000 ejecuciones hipotecarias en toda la nación que han dejado cientos de miles de familias en la calle y con una deuda de por vida. Lo dramático del asunto estriba en que detrás de las cifras hay personas, vivencias y proyectos que se truncan; sueños que se convierten en la peor de las pesadillas. Las Naciones Unidas ha condenado recientemente la política de vivienda del Estado español como una de las peores del mundo. Raquel Rolnic relatora de la ONU, basada en la existencia de millones de casas vacías y en el aumento estrepitoso de los embargos, ha denunciado que la legislación hipotecaria española es incompatible con la normativa básica europea. A la par de estos embargos se están dando continuos casos de suicidio de personas honestas que lo han perdido todo. Esta situación ha prendido las alarmas sociales y ha

23. StéphaneHessel (1917-2013) fue un diplomático, escritor y político francés que contribuyó a la redacción de la Declaracion Universal de los Derechos Humanos en 1948.

24. Ada Colau es la cara más visible de esta asociación. Es su portavoz y una de las impulsoras del colectivo; lleva una década luchando por el derecho a la vivienda. 
merecido una amplia difusión en los medios de comunicación y en las redes sociales, ejerciendo con ello una fuerte presión al gobierno para que reaccione y tome las medidas justas.

Ada Colau ${ }^{25}$ como portavoz del movimiento PAH afirma que para que se dé una política realmente transformadora se necesitan procesos colectivos en los que participe el máximo de ciudadanos. Argumenta la existencia de una crisis de la democracia representativa y un desfasamiento de los partidos políticos que no les permite responder a la realidad actual. Lo primero que tendría que hacerse es eliminar esta relación obscena de dependencia entre el poder político y el económico que lleva a un sometimiento absoluto del primero a los dictados del segundo y a una primacía de los intereses de los grandes poderes económicos que se hace más que evidente en el drama de las hipotecas. Los dos partidos mayoritarios (el Psoe y el PP) se han dedicado a bloquear cualquier tipo de solución dada su relación de dependencia del poder financiero. Mientras esto sea así y exista esa puerta giratoria en la que la gente pasa por la política y acaba en un consejo de administración la democracia plena no será posible. Sin duda, esta crisis económica señala como principal responsable a la "especulación financiera" que por supuesto, casi nunca tiene un nombre y un rostro. A lo sumo se mencionan muy vagamente los fondos de cobertura, pero en la mayoría de los casos las operaciones especulativas se permiten legalmente o se eluden tan fácilmente las normas que las regulan que el juego vale la candela. En realidad, estamos frente a una pasión particular de los financieros por los juegos de azar alimentada por el hecho de que las finanzas en sí mismas tienen la dinámica de un juego y como suele suceder a veces, el jugador con problemas hace trampa. La ley del juego es contundente: "Si usted no lo hace lo hará otro".

La concentración de la riqueza es el resultado de un crecimiento anormal de las finanzas en los últimos treinta años, pero el elemento clave son las consecuencias fiscales de la movilidad del capital permitido desde los años ochenta. Nos referimos al fenómeno de los paraísos fiscales: ${ }^{26}$ Andorra, Antillas Neerlandesas, Aruba, Suiza, Suecia, Emiratos Árabes Unidos, Chipre, Hong Kong, Bahamas, Fiji, República de Malta, Jamaica, Panamá, Singapur, Islas Vírgenes Británicas, República Libanesa, Gran Ducado de Luxemburgo y el Principado de Mónaco, entre otros, que cobran un impuesto muy bajo sobre el capital para de este modo atraer inversionistas de todo el mundo. Es ahí donde se ha levantado una especie de suspensión de la soberanía fiscal de los países frente al "capital sin patria" que viaja con el pasaporte de un nuevo Estado cosmopolita sin territorio que llamaremos "Ricolandia", una suerte de poder colonial que ha reducido impuestos estatales al resto del mundo.

La comercialización de la democracia es uno de los principales signos de identidad de la Europa en crisis. Desde hace décadas gobiernos y políticas de signo neoliberal parecen haberse convertido en subordinados de los grandes poderes económicos que compiten de manera desleal por obtener los máximos beneficios electorales. En la UE la democracia funciona como un mercado político en el que los electores compran los programas electorales como si fuesen mercancías y obviamente escogen la que mejor satisfaga sus intereses egoístas. Los candidatos políticos actúan como proveedores de servicios que intentan cautivar al electorado a fin de acumular poder mediante el voto. De esta forma los intereses privados se imponen sobre las virtudes colectivas y la democracia solo sirve para optimizar los beneficios particulares.

La comercialización de la política y de la democracia en la época de la globalización se manifiesta de múltiples maneras. Uno de los principales aspectos a tener en cuenta radica en que las campañas electorales políticas y su publicidad son pagadas por empresas privadas, hecho que convierte a los partidos en lacayos del poder económico. Otra de las fachadas de esta ficción de democracia es la

25. Ada Colau http://www.eldiario.es/catalunya/Entrevista-Ada_Colau-Plataforma_de_Afectados_por_la_Hipoteca-desahuciosILP_hipotecaria_0_69643131.html

26. Un paraíso fiscal es un país que exime del pago de impuestos a los inversores extranjeros que mantienen cuentas bancarias o constituyen sociedades en su territorio. Mientras los ciudadanos y empresas residentes en el propio país están obligados al pago de sus impuestos como en cualquier otro lugar del mundo, los extranjeros gozan en la mayoría de los casos de una exención total, o al menos de una reducción considerable de los impuestos que deben pagar. Esto es así siempre y cuando no realicen negocios dentro del propio paraíso fiscal. 
compraventa de votos con dinero público o privado, una de las formas más bajas de corrupción ya que la banalización del voto hace que se pierda la capacidad real de elegir. El dominio de la economía sobre la política es tan grande que el derecho al voto termina siendo el derecho a elegir partidos marioneta que solo representan los intereses de la super aristocracia financiera. Sin duda la lucha de los nuevos movimientos sociales del siglo XXI se enfocará a que la pequeña y frágil barca de la democracia pueda anclar en nuevos puertos sin naufragar en el intento.

\section{Conclusiones}

En estos momentos el optimismo en Europa se ha desvanecido y congelado la sonrisa en la cara de políticos y economistas. El riesgo de que esta recesión económica pueda desembocar en una grave depresión económica como la Gran Depresión de $1929^{27}$ es tal que nadie se atreve a decir que esta crisis llegó a su fin. Es cierto que después de la crisis del treinta hemos conocido otras que afectaron a Japón y a los mismos EE UU como las que se presentaron en 1985 y 1987. Pero Europa no ha salido indemne. Entre 1994 y el 2001 se registró una repetición de la crisis que afectó las economías de Asia, Estados Unidos, Rusia, Argentina y Japón; sin embargo, ninguna de ellas tuvo la intensidad, la persistencia y la generalización que parece tener la presente aún en pleno desarrollo.

Jean Ziegler ${ }^{28}$ tras analizar la situación económica dominante en el mundo recomienda "ocupar masivamente los bancos, nacionalizarlos y confiscar las arrogantes riquezas robadas por los especuladores financieros". Vivimos, según Ziegler, en un "orden mundial criminal y caníbal" en el que los especuladores financieros deciden de forma legal (las leyes y las principales instituciones están al servicio de la superaristocracia financiera) quién va a morir de hambre y quién no ${ }^{29}$. De hecho, la crisis tiene unos culpables y responsables que hay que denunciar, auditar y juzgar ante un tribunal similar al de Nüremberg. No se puede entender cómo casi una quinta parte de los seres humanos sufre y muere de hambre si se cuenta con los recursos agroalimentarios suficientes para alimentar al doble de la población mundial actual. Las crisis de España, Italia y Grecia son graves, pero nos llama a una toma de conciencia. La gente quiere saber cómo es posible que se llegue a una situación así y si progresa en esta toma de conciencia descubrirá que los hambrientos de África y el sufrimiento que se está tomando a Europa tienen un denominador común: la oligarquía financiera multinacional. Necesitamos una insurrección de la conciencia para acabar con la dictadura mundial del capital financiero y conformar un frente común de solidaridad y de resistencia transcontinental. El Che Guevara decía: "Los muros más sólidos se quiebran por sus fisuras". Ya se ven fisuras en todas partes ${ }^{30}$.

El movimiento social americano Occupy Wall Street lanzó la consigna "somos el 99 \% contra el $1 \%$ ” para decir que el mundo de las finanzas es solo una ínfima minoría de la población mundial. Como todas las consignas, esta no tiene la intención de declarar una verdad estadística sino de dar una imagen de la situación. En efecto, la llamada superaristocracia financiera es solo una milésima parte de la población mundial, pero la otra cara de la moneda establecida por el lema son las masas desheredadas de África, Asia y América Latina y las que ahora entrarán a formar parte los países de la UE. Citando a Dilma Rousseff, Presidenta de Brasil:

Nosotros ya hemos vivido esto. El FMI nos impuso un proceso que llamaron de ajuste, ahora lo llaman austeridad. Había que cortar todos los gastos, los

27. La Gran Depresión fue una recesión económica mundial que empezó en 1929 y se extendió hasta casi los años cuarenta. Fue considerada la más importante crisis de la historia moderna. La Gran Depresión tuvo efectos devastadores tanto en los países desarrollados como en desarrollo. El comercio internacional se vio profundamente afectado al igual que los ingresos personales, los ingresos fiscales, los precios y los beneficios empresariales. Ciudades de todo el mundo resultaron gravemente afectadas especialmente las que dependían de la industria pesada. La construcción prácticamente se detuvo en muchos países.

28. Sociólogo y político suizo vicepresidente de Derechos Humanos de la ONU y autor, entre otros, del recomendable libro Destrucción masiva. Geopolitica del hambre (Península)

29. Es el escándalo del siglo. Algunas cifras de la FAO del 2011 indican que cada cinco segundos un niño de menos de diez años muere de hambre. Más de mil millones de los casi siete mil millones de habitantes del planeta sufren permanentemente de subnutrición. La FAO expresa que la agricultura mundial podría alimentar a doce mil millones de personas, casi el doble de la humanidad. Un nińo que muere de hambre hoy es un asesinato. http:/www.20minutos.es/noticia/1487418/0/ziegler/entrevista/hambre/

30. JeanZiegler http://www.20minutos.es/noticia/1487418/0/ziegler/entrevista/hambre/ 
corrientes y los de inversión. Aseguraban que así llegaríamos a un alto grado de eficiencia, los salarios bajarían y se adecuarían los impuestos. Ese modelo llevó a la quiebra de casi toda Latinoamérica en los años ochenta ${ }^{31}$.

Las protestas sociales no van a faltar en la Europa del futuro como ya se ha visto en Londres, Francia, España, Italia, Grecia y Portugal, y seguirán ofreciendo a la información oficialista la oportunidad para reducir el descontento a una cuestión de orden público como si los vándalos no fueran esa superaristocracia financiera con su insoportable avaricia que está arrebatando a gente honesta de todo el mundo su trabajo, su vida, sus familias, su dignidad y su futuro.

Se debe iniciar un plan político de trabajo para fortalecer el bienestar social y reequilibrar la distribución de la riqueza. Los partidos políticos europeos deben comprender que el gran desafío es eliminar el vínculo de dependencia entre economía capitalista y democracia que como un cáncer amenaza las instituciones democráticas. El poder debe ser entregado al gobernante legítimo elegido por su pueblo y no se debe correr el riesgo de poner millares de vidas en manos de un escurridizo y peligroso poder oscuro. No se debe perder de vista que las crisis no son eventos objetivos e independientes de la voluntad humana y su desarrollo y resultados no están escritos en el libro del destino, sino que dependen de las decisiones humanas. De las crisis se puede salir; lo importante es tomar la dirección correcta. Si se tiene que ir de Milán a Lisboa no se debe tomar el tren a Budapest. Y para terminar, no perder de vista que la inundación de liquidez no es precisamente la cura de este mal.

\section{Bibliografía}

- BAUMAN, Z ( 2003) Modernidad líquida. Editorial Fondo de Cultura Económica. México D.F.

- BALDWIN, R.NAVARETTI,G.BOERI,T. (2007) Come sta cambiando l'Italia. Il mulino. Bologna.

- BOERI,T. Garibaldi,P. (2008). Un nuovo contratto per tutti. Editore Chiarelet. Milano.

- HESSEL, S (2011) Indignatevi. Add Editores. Torino

- INGRAO, P(2011) Indignarsi non basta. Aliberti Editore.Roma

- IEZZI, M y MASTROBUONI, T (2010) Gioventù sprecata. Perchè in italia si faticaa diventare grande. Editori Laterza. Bari Italia.

- GIANNULI, A (2012) Uscire dalla crisi è possibile. Adriano Salani Editore.Milano.

- COLOMBO, A (2010) La disunità del Mondo. Feltrinelli, Milano.

- HUNTINGTON, S (1997) Lo scontro di civilità e il nuevo ordine mondiale. Garzanti, Milano

- FUKUYAMA, F (1992) La fine della storia e l’ultimo uomo. Rizzoli, Milano.

- ISTAT (2009) Difficoltà nella tranzicione dei giovani allo stato adulto e crecità nei percorsi di vita femminile. Istat Roma

- PERROTI, R (2008) L'università truccata. Einaudi, Torino

- NEGRI, A y HARDT, M (2002) Impero: Il nuovo ordine della globalizzazione. Rizzoli, Bergamo.

\section{Webgrafía}

- (www.istat.it/salastampa/comunicati/non_calendario/20090714_01/testointegrale20090714.pdf).

31. Con estas palabras de la presidenta de Brasil Dilma Rousseff, comienza el informe Crisis, desigualdad y pobreza publicado por Intermón Oxfam. http://www.sociologossinfronteras.org/wp-content/uploads/2013/01/CRISIS-desigualdad-y-pobreza-IntermonOxfam1.pdf 
- http://www.attac.es/2013/02/08/no-debemos-resignarnos-sino-indignarnos-tomar-la-calle-y-las-sedes-de-losdefraudadores/

- http://www.referendumlavoro.it/index.php?option=com_content\&view=article\&id=94:non-si-esce-dalla-crisiaumentando-lorario-e-riducendo-ancora-loccupazione\&catid=13:riflessioni\&Itemid= 51

- http://www.acabaconlacrisis.es/

- http://www.ucm.es/info/nomadas/19/avrocca2.pdf

- http://www.sociologossinfronteras.org/wp-content/uploads/2013/01/CRISIS-DESIGUALDAD-Y-POBREZA-Intermon-Oxfam1.pdf

$162 \varangle$ Universidad de San Buenaventura, Cali - Colombia 